\title{
A discrete choice task to measure preferences for harsh discipline among parents of young children
}

\author{
Eric P. Green ${ }^{\mathrm{a}, *}$, Rhea M. Chase ${ }^{\mathrm{b}}$, John Zayzay ${ }^{\mathrm{c}}$, Amy Finnegan ${ }^{\mathrm{a}}$, Eve S. \\ Puffer ${ }^{\mathrm{a}, \mathrm{d}}$ \\ ${ }^{a}$ Duke Global Health Institute, Box 90519, Durham, North Carolina 27708, USA \\ ${ }^{b}$ Judge Baker Children's Center, 53 Parker Hill Avenue, Boston, MA 02120, USA \\ ${ }^{c}$ International Rescue Committee, Monrovia, Liberia \\ ${ }^{d}$ Duke University, Department of Psychology and Neuroscience, Box 90086, 417 Chapel \\ Drive, Durham, NC 27708, USA
}

\footnotetext{
*Please address correspondence to Eric Green, (919)681-7289

Email addresses: eric.green@duke.edu (Eric P. Green), rchase@jbcc.harvard.edu (Rhea M. Chase), john.zayzay@rescue.org (John Zayzay), amy.finnegan@duke.edu (Amy Finnegan), eve.puffer@duke.edu (Eve S. Puffer)

${ }^{1}$ For their assistance on this study, the authors would like to thank Stephanie Banks, Joanne Creighton, Esther Karnley, Anjuli Shivshanker, Crystal Stewart, Bangalie Trawally, and the staff of the International Rescue Committee in Monrovia, Liberia.
} 


\begin{abstract}
Maltreatment in early childhood is difficult to measure. Self-report surveys of parents and guardians are the most common method used, but in many settings social desirability may lead to underestimates of prevalence. There is also reason to be concerned about response bias in the context of intervention trials. To diversify the tools available to intervention researchers, we created and tested a discrete choice experiment to elicit parent preferences for harsh discipline. This study was conducted in Liberia as part of a randomized controlled trial of a positive parenting program. Baseline data were collected from 609 parents and guardians living in Monrovia and caring for a child age 3 or 4 . Participants completed a discrete choice experiment that consisted of 12 parenting vignettes, in addition to a survey of parenting attitudes and behaviors. The vignettes were presented to parents as digital comic strips. Each scene could vary on four attributes: child gender; child offense; setting; number of adults present. For each scene, participants selected 1 of 5 discipline strategies that they would in that situation. The visual discrete choice task was easy to implement, well understood by participants, and has evidence of construct validity as a measure of parent preferences for harsh discipline. Tools like this expand the options for researchers studying the maltreatment of pre-school age children, particularly in the context of program evaluations where post-intervention observations may be at increased risk of response bias. It may also be useful in a clinical context.
\end{abstract}

Keywords: $\quad$ parenting, harsh discipline, response bias, discrete choice, Liberia, global

mental health 


\section{Introduction}

Maltreatment in early childhood is difficult to measure, posing challenges for psychologists in both assessment and treatment. In high-income countries, administrative records are often used to estimate population-level incidence and prevalence of abuse (Fallon et al., 2010). Throughout much of the world, however, routine data on child abuse are not collected. Estimates are limited to periodic national surveys, such as the Demographic and Health Surveys (DHS) and the Multiple Indicator Cluster Surveys (MICS), and to smaller studies of non-representative samples (Child Protection Monitoring and Evaluation Reference Group, 2014). Most surveys like the DHS and MICS rely on parent or guardian (i.e., perpetrator) retrospective self-report. While some instruments have child (i.e., victim) report modules (WHO, 2013; Zolotor et al., 2009), none are designed for assessing pre-school age children.

There is ample debate and disagreement about how to best define maltreatment, but these self-report surveys share one thing in common: high face validity. It is obvious to the respondent that the survey enumerator is asking about child abuse. As such, social desirability should work to underestimate the true prevalence of maltreatment in settings where there are strong norms against harsh discipline. In places where harsh discipline practices like beating are more normative (Lansford et al., 2005), however, the potential for response bias may be lower. For example, across 16 countries in West and Central Africa surveyed by the DHS and MICS programs between 2006 and 2014, nearly 9 out of 10 parents reported using discipline strategies categorized as physical 
punishment or psychological aggression (UNICEF, 2013).

Regardless of the setting, there is reason to be concerned about response bias in the context of intervention trials. Teaching parents about the negative effects of maltreatment on child development is an explicit aim of many evidence-based positive parenting interventions (Kaminski et al., 2008). Therefore, even in places where harsh punishment is more normative, an effective parenting program should increase the likelihood of response bias among the treatment group as parents learn that their behavior is viewed by the program as potentially damaging to children. Without corroborating evidence from other informants or methods, post-intervention reductions in self-reported harsh discipline may reflect an impact on parents' social awareness of the undesirable nature of certain parenting behaviors rather than an impact on actual parenting behavior.

It is partly for this reason that researchers emphasize the importance of developing complementary methods of detecting outcomes. Direct observation is promising approach for validating parent-report data (Aspland and Gardner, 2003), but few parenting trials have incorporated observational measures of parent and child behavior (Gardner et al., 2016). It may be that the time and resources required to adapt observational measures for new contexts, establish the inter-rater reliability of observers, and code recordings of interactions imposes too high a burden.

In an effort to diversify the tools available to intervention researchers, we created and tested a discrete choice experiment to elicit parent preferences 
for harsh discipline. We believe that this parent-report instrument designed for low-literacy populations has less obvious demand characteristics than traditional survey instruments and can be a useful complement in intervention trials. Our findings support the feasibility of implementation and the construct validity of the method.

\section{Methods}

\section{Setting and Participants}

This study was conducted in Liberia as part of a randomized controlled trial of a positive parenting program called "Parents Make the Difference". The data presented here come from baseline surveys of parents or guardians of children

ages 3 or 4 years who enrolled in the program. Parents were recruited from three urban settlements in greater Monrovia. Adults 18 years and older were eligible to enroll if they were a primary caregiver for a child in the eligible age range, and if both parent and child were residing in the community. Data were collected before participants were randomized to immediate (treatment) or delayed (control) program entry.

\section{Procedures}

Liberian enumerators (male and female) surveyed each parent individually at their home. Surveys were conducted on 7-inch Android tablets running 
ODK Collect. Enumerators read each prompt aloud and recorded participant responses on the tablets. Data collection took place in three waves from July 2014 to July 2016.

\section{Measures}

\section{Discrete Choice Experiment}

We designed a modified discrete choice experiment (DCE) to elicit parent preferences for harsh discipline. DCEs have been used in various fields like marketing and health care to understand consumer preferences when data on actual decision making (i.e., revealed preferences) is not available (Mangham et al., 2009). In a typical DCE, participants are repeatedly presented with pairs of scenarios that vary on several relevant attributes and asked to decide between the two choice sets. We modified this approach by presenting one scenario at a time and asking parents to select 1 of 5 discipline strategies in response to each scenario.

We asked participants to review digital illustrations depicting a child misbehaving (see Figure 1 for an example). Each scene could vary on four attributes: child gender (boy, girl); child offense (spilling drink, whining, kicking parent); setting (home, market); number of adults present (one, two). For each scene, participants indicated how they would respond if they were the parent in the story. Response options were presented as a set of five drawings: (A) time out; (B) beating; (C) discussing; (D) yelling; and (E) ignoring (see 
Figure 2). Collectively, there were 24 permutations of attributes and levels, but we used a fractional factorial design (orthogonal and balanced) that limited the set to 12 different comic strips. With 609 participants, this resulted in 7,308 data points. The activity took approximately 15 minutes to explain and administer.

[Figure 1 about here.]

[Figure 2 about here.]

Prior to starting the activity, enumerators read the following prompt: "Now we are going to do something different. I will show you some picture stories of children and parents, ask you what is happening in the pictures, and then ask you what you would do if you were the parent." Enumerators then showed participants three example comic strips, one at a time, and asked "What is happening?" for each one. If participants did not understand, the enumerators explained the comic strip. The enumerators then showed participants the response options and asked "What is happening in each scene?", clarifying for participants as needed. When the practice session concluded, enumerators read the following prompt and showed the first of 12 comic strips: "Now I will show you 12 different picture stories. After each one, I will ask you to tell me what you would do if you were the parent in the story. There are no right or wrong answers."

We constructed a harsh discipline preference (HDP) score by scoring each response of "beat" or "yell" as (1) and summing across all 12 scenarios. 


\section{Survey Instrument}

As this study was part of a larger baseline assessment, we also administered a survey to every parent that included questions about the following domains: household composition and demographics; knowledge, attitudes, and beliefs about parenting and child development; discipline practices; and family, parent, and child well-being. All items were adapted for the Liberian English vernacular by the local study team prior to cognitive interviewing with 109 adults not involved in the trial. Survey items about parenting followed the DCE.

Discipline Interview. We adapted 9 items from the Discipline Interview (DI; Lansford et al., 2005) that ask parents about the frequency of harsh discipline strategies. Enumerators told parents: "All children do bad things sometimes. I will say things parents can do when children do bad things. Tell me how many times you do the thing I say." Parents responded to questions like, "How frequently do you spank, slap, or hit your child," on a 4-point scale from "never" (1) to "often" (4). We constructed a harsh discipline scale score by averaging responses to the set of items $(\alpha=0.83)$.

Parental Acceptance and Rejection Questionnaire. We administered 24 items from the short form of the Parental Acceptance and Rejection Questionnaire (PARQ; Rohner and Khaleque, 2008). Enumerators told parents: "I will say things about how you are with your child. Tell me how true that thing is: almost always true, sometimes true, rarely true, or almost never true." 
Parents used this 4-point scale to respond to questions like, "I say nice things about my child" and "I hit my child even when (s)he may not deserve it". We followed the original reverse scoring instructions but constructed the scale as an average rather than a sum score $(\alpha=0.75)$.

\section{Statistical Analysis}

We ran cross tabulations to verify participant comprehension of the discrete choice task and fit a multilevel logistic regression with a random effect for individuals to account for repeated measures to confirm that our manipulation of the child offense attribute was successful. We then described the new HDP scale, examined correlations with other measures to assess convergent validity, and used an independent samples t-test to assess discriminant validity. These final analyses were conducted on a subset of 408 participants from the second and third communities enrolled in the study as we did not administer a full baseline to parents from the first community.

\section{Ethical Review}

This study protocol was approved by institutional review boards at [OMITTED FOR REVIEW] and the University of Liberia. Enumerators obtained written informed consent from parents prior to participation. 


\section{Results}

\section{Participant Characteristics}

609 parents participated in the baseline assessment. Participant characteristics are summarized in Table 1. Participants were mostly women with little formal education. Almost half reported that they are married or living with a partner. The distribution of economic status in the sample reflected the distribution observed for Monrovia in the 2011 Liberia Malaria Indicator Survey (National Malaria Control Program et al., 2012).

[Table 1 about here.]

\section{Comprehension Check}

The majority of participants understood all three example comic strips without enumerator assistance or only needed a small clarification (71.4\%). A smaller share of participants needed more substantial coaching before the enumerator could move forward (25.0\%), and 22 participants never demonstrated sufficient understanding according to the enumerators (or had missing practice data) and were dropped from further analysis (3.6\%), resulting in an analysis sample of 587 parents. 


\section{Manipulation Check}

Table 2 displays the results of a multilevel logistic regression of parents' discipline choices on a vector of comic strip attributes, including an indicator of whether the offense depicted in the scene was major (i.e., kicking the parent). The odds of choosing harsh discipline-yelling or beating, rather than talking, time out, or ignoring - increase by a factor of 1.28 (1.14 to 1.44) when moving from a minor offense (i.e., spilling a drink, whining) to a major offense (i.e., kicking the parent). This is evidence that manipulation of offense was successful.

[Table 2 about here.]

\section{Description of Harsh Discipline Preference Score}

On average, participants selected a harsh discipline strategy in 4.2 out of 12 scenarios $(S D=3.2)$. Figure 3 displays a density plot of the distributions of normalized scale scores. The median value of the new HDP scale was within 10.0 percent of the median value of the Discipline Interview scale score.

[Figure 3 about here.] 


\section{Construct Validity of Harsh Discipline Preference Score}

\section{Convergent Validity}

The new HDP score correlates 0.26 with the Discipline Interview items and 0.37 with the PARQ items.

\section{Discriminant Validity}

We assess discriminant validity by creating a dichotomous indicator of corporal punishment that is set to 1 if the participant responded "almost always" to PARQ16 "I hit my child even when (s)he may not deserve it" OR "often" to DI5 "How frequently do you spank, slap, or hit your child?" Parents who reported engaging in either of these behaviors $(25.2 \%)$ expressed stronger preferences for harsh discipline $(M=4.5, S D=3.7)$ compared to parents who did not report this behavior $(M=3.7, S D=3.1), t(404)=-2.2, p<0.05$.

\section{Discussion}

We found that the visual discrete choice task was easy to implement, well understood by participants, and has evidence of construct validity as a measure of parent preferences for harsh discipline. Tools like this expand the options for researchers studying the maltreatment of pre-school age children, particularly in the context of intervention trials where post-intervention observations may be at increased risk of response bias. It may also be useful in a clinical 
context. As child maltreatment is a risk and complicating factor for emotional and behavioral disorders, as well as a safety concern in and of itself, professionals providing mental healthcare need valid and reliable tools for assessing maltreatment that are efficient and valid with minimal bias.

In this study, we presented scenes as digital comic strips on a tablet computer as part of a larger assessment battery, but electronic administration is not necessary; it would have been feasible to print each scenario for paper administration. We also decided to have a trained Liberian enumerator administer the activity and record participant responses, but the visual nature of this task is well-suited for self-administration with or without a live demonstration and practice session. Presumably self-administration would further reduce the possibility for response bias due to increased privacy. Future research should explore alternate methods of administration and test materials that blend an assessment of preferences toward harsh punishment with other, non-sensitive topics to further reduce demand characteristics.

Liberia was a good setting to explore the construct validity of this approach because self-reported harsh discipline of children is high (UNICEF, 2013). A limitation is that we were unable to assess criterion validity as we lacked a gold-standard measure of child maltreatment. Future work should evaluate how well this method measures response to treatment. 


\section{References}

Aspland, H., Gardner, F., 2003. Observational measures of parent-child Interaction: An introductory review. Child and Adolescent Mental Health 8 (3), 136-143.

Child Protection Monitoring and Evaluation Reference Group, 2014. Measuring violence against children: Inventory and assessment of quantitative studies. Tech. rep., UNICEF.

Fallon, B., Trocmé, N., Fluke, J., MacLaurin, B., Tonmyr, L., Yuan, Y.Y., 2010. Methodological challenges in measuring child maltreatment. Child Abuse \& Neglect 34 (1), 70-79.

Gardner, F., Montgomery, P., Knerr, W., 2016. Transporting evidence-based parenting programs for child problem behavior (age 3-10) between countries: Systematic review and meta-analysis. Journal of Clinical Child \& Adolescent Psychology 45 (6), 749-762.

Kaminski, J. W., Valle, L. A., Filene, J. H., Boyle, C. L., 2008. A meta-analytic review of components associated with parent training program effectiveness. Journal of Abnormal Child Psychology 36 (4), 567-589.

Lansford, J. E., Chang, L., Dodge, K. A., Malone, P. S., Oburu, P., Palmérus, K., Bacchini, D., Pastorelli, C., Bombi, A. S., Zelli, A., Tapanya, S., Chaudhary, N., Deater-Deckard, K., Manke, B., Quinn, N., 2005. Physical discipline and children's adjustment: Cultural normativeness as a moderator. Child Development 76 (6), 1234-1246. 
Mangham, L. J., Hanson, K., McPake, B., 2009. How to do (or not to do)...designing a discrete choice experiment for application in a low-income country. Health Policy and Planning 24 (2), 151-158.

National Malaria Control Program, Ministry of Health and Social Welfare, Liberia Institute of Statistics and Geo-Information Services, ICF International, 2012. Liberia malaria indicator survey 2011.

Rohner, R., Khaleque, A. (Eds.), 2008. Handbook for the Study of Parental Acceptance and Rejection, 4th Edition. Rohner Research Publications.

UNICEF, 2013. A Statistical Snapshot of Child Protection in West and Central Africa. Tech. rep.

WHO, C., 2013. 2013 GSHS Data User's Guide. Tech. rep.

Zolotor, A. J., Runyan, D. K., Dunne, M. P., Jain, D., Péturs, H. R., Ramirez, C., Volkova, E., Deb, S., Lidchi, V., Muhammad, T., Isaeva, O., 2009. ISPCAN Child Abuse Screening Tool Children's Version (ICAST-C): Instrument development and multi-national pilot testing. Child Abuse \& Neglect $33(11), 833-841$. 


\section{List of Figures}

1 Example comic strip. In this comic strip, the NUMBER OF ADULTS PRESENT is 2, the child GENDER is male, the OFFENSE is kicking, and the SETTING is the market. The gender of the main caregiver in the story did not vary experimentally. Instead, the gender of the main caregiver character was matched to the gender of the participant. This particular strip would only have been presented to men. . . . . . . . . . . . . . .

2 Choice experiment response options. For each scene, participants indicated how they would respond if they were the parent in the story. Response options were presented as a set of five illustrations: (A) time out; (B) beating; (C) discussing; (D) yelling; and (E) ignoring. Participants could also answer "Don't Know/Refuse to Answer". . . . . . . . . . . . . . . . .

3 Density plot of distributions of normalized scale scores. Median scores indicated with vertical labels. . . . . . . . . . . . . 

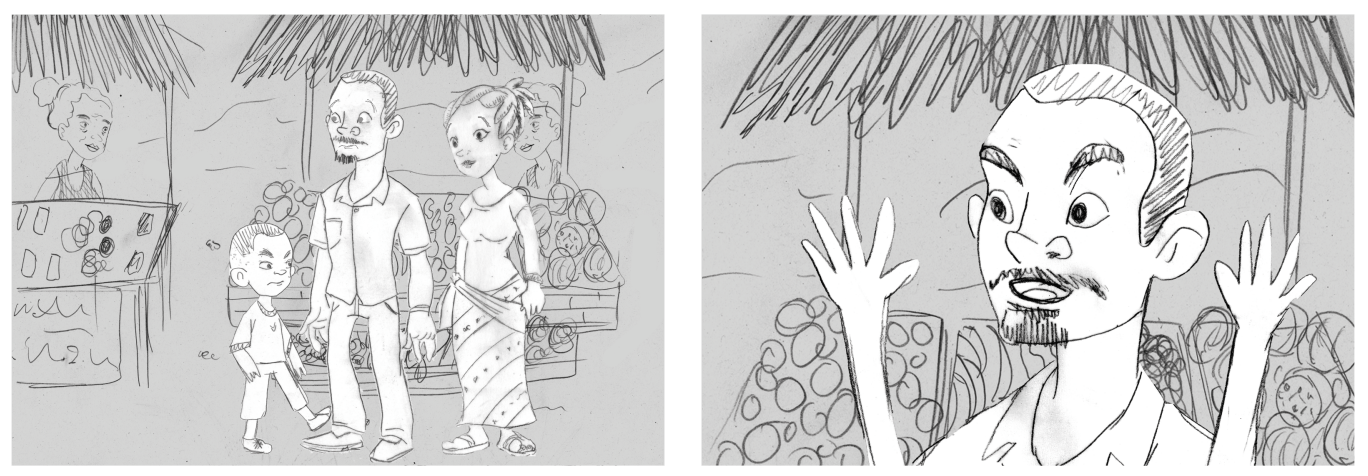

Figure 1: Example comic strip. In this comic strip, the NUMBER OF ADULTS PRESENT is 2, the child GENDER is male, the OFFENSE is kicking, and the SETTING is the market. The gender of the main caregiver in the story did not vary experimentally. Instead, the gender of the main caregiver character was matched to the gender of the participant. This particular strip would only have been presented to men. 


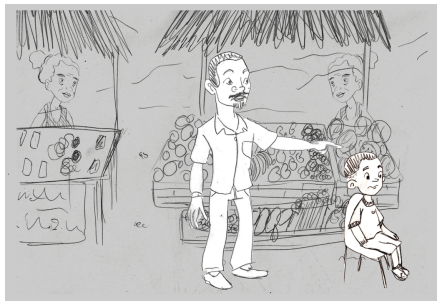

A

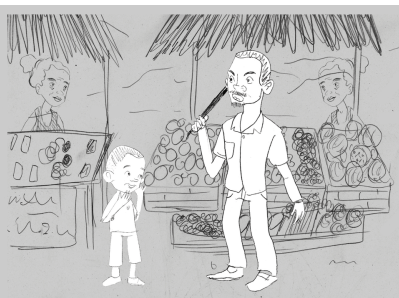

B

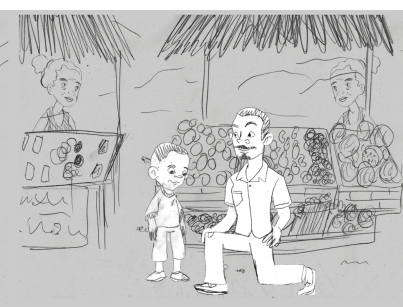

C

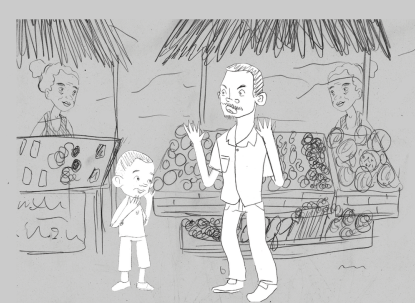

D

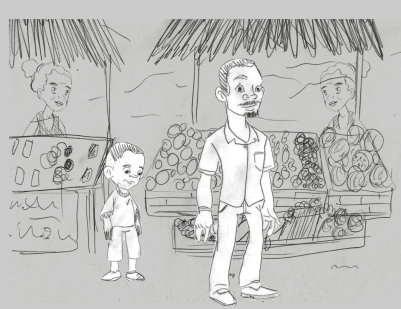

E

Figure 2: Choice experiment response options. For each scene, participants indicated how they would respond if they were the parent in the story. Response options were presented as a set of five illustrations: (A) time out; (B) beating; (C) discussing; (D) yelling; and (E) ignoring. Participants could also answer "Don't Know/Refuse to Answer". 


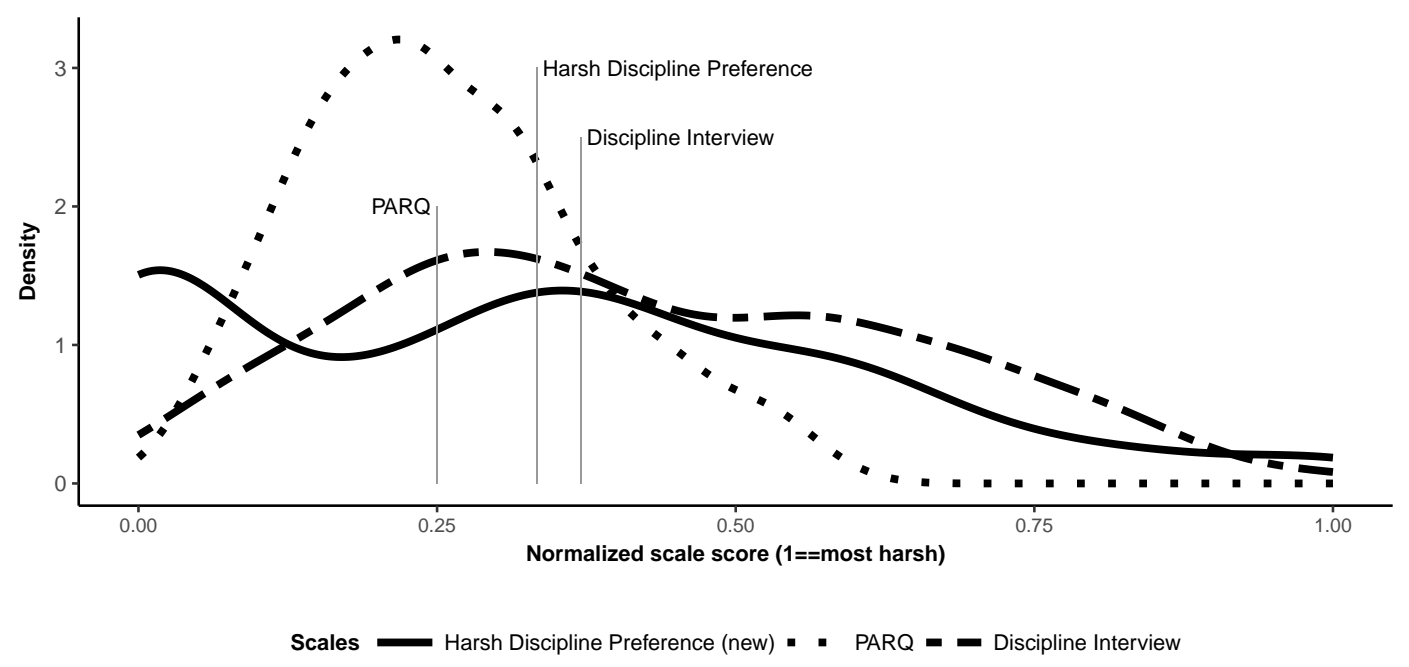

Figure 3: Density plot of distributions of normalized scale scores. Median scores indicated with vertical labels. 
Table 1: Participant characteristics

\begin{tabular}{lr}
\hline Variables & Baseline \\
\hline$N$ & 609 \\
Mean Age $(S D)$ & $33.3(10.2)$ \\
Female (\%) & 91.5 \\
Completed primary (\%) & 8.2 \\
Married or cohabiting (\%) & 47.1 \\
\hline
\end{tabular}


Table 2: Multilevel logistic regression of discipline choice on a vector of scene attributes

\begin{tabular}{lr}
\hline & Dependent variable: \\
\cline { 2 - 2 } & Parent selects harsh discipline \\
\hline Intercept & $0.38(0.32$ to 0.46$)$ \\
Kicked parent & $1.28(1.14$ to 1.44$)$ \\
Child is boy & $1.11(0.98$ to 1.24$)$ \\
At home & $0.93(0.83$ to 1.05$)$ \\
Two parents present & $0.89(0.79$ to 0.99$)$ \\
\hline Random effect variance & 2.09 \\
Observations & 7,039 \\
Parents & 587 \\
\hline
\end{tabular}

